\title{
Application of fixed point theorem in probabilistic metric space to quicksort algorithm
}

\author{
Judit Robu, Anna Soós \\ Babes-Bolyai University, Faculty of Mathematics and Computer Science \\ robu@cs.ubbcluj.ro, asoos@math.ubbcluj.ro
}

\begin{abstract}
Our aim in this work is to prove the existence and uniqueness of solution for recurrence equations associated to the asimptotic complexity analysis of Quicksort algorithms using some results from the theory of probabilistic metric spaces.
\end{abstract}

Keywords: fixed points, probabilistic metric space, Quicksort Algorithm MSC: 60G57, 28A80, 68W40

\section{Preliminaries}

The theory of probabilistic metric spaces, introduced in 1942 by K. Menger [5], and was developed by numerous authors [7].

A mapping $F: \mathbb{R} \rightarrow[0,1]$ is called a distribution function if it is non-decreasing and left continuous.

By $\Delta$ we shall denote the set of all distribution functions $F$. Let $\Delta$ be ordered by the relation " $\leq$, i.e. $F \leq G$ if and only if $F(t) \leq G(t)$ for all real t. Also $F<G$ if and only if $F \leq G$ but $F \neq G$.

We set $\Delta^{+}:=\{F \in \Delta: F(0)=0\}$.

$H$ will denote the Heviside distribution function defined by

$$
H(x)= \begin{cases}0, & x \leq 0 \\ 1, & x>0\end{cases}
$$

Let $X$ be a nonempty set. For a mapping $\mathcal{F}: X \times X \rightarrow \Delta^{+}$and $x, y \in X$ we shall denote $\mathcal{F}(x, y)$ by $F_{x, y}$, and the value of $F_{x, y}$ at $t \in \mathbb{R}$ by $F_{x, y}(t)$, respectively.

The pair $(X, \mathcal{F})$ is a probabilistic metric space if $X$ is a nonempty set and $\mathcal{F}: X \times X \rightarrow \Delta^{+}$is a mapping satisfying the following conditions: 
$1^{0} F_{x, y}(t)=F_{y, x}(t)$ for all $x, y \in X$ and $t \in \mathbb{R}$;

$2^{0} F_{x, y}(t)=1$, for every $t>0$, if and only if $x=y$;

$3^{0}$ if $F_{x, y}(s)=1$ and $F_{y, z}(t)=1$ then $F_{x, z}(s+t)=1$.

A mapping $T:[0,1] \times[0,1] \rightarrow[0,1]$ is called a $t$-norm if the following conditions are satisfied:

$4^{0} T(a, 1)=a$ for every $a \in[0,1]$;

$5^{0} T(a, b)=T(b, a)$ for every $a, b \in[0,1]$;

$6^{0}$ if $a \geq c$ and $b \geq d$ then $T(a, b) \geq T(c, d)$;

$7^{0} T(a, T(b, c))=T(T(a, b), c)$ for every $a, b, c \in[0,1]$.

We list here the simplest:

$T_{1}(a, b)=\max \{a+b-1,0\}$,

$T_{2}(a, b)=a b$,

$T_{3}(a, b)=\operatorname{Min}(a, b)=\min \{a, b\}$,

A Menger space is a triplet $(X, \mathcal{F}, T)$, where $(X, \mathcal{F})$ is a probabilistic metric space, $T$ is a t-norm, and instead of $3^{0}$ we have the stronger condition:

$8^{0} F_{x, y}(s+t) \geq T\left(F_{x, z}(s), F_{z, y}(t)\right)$ for all $x, y, z \in X$ and $s, t \in \mathbb{R}_{+}$.

The $(t, \epsilon)$-topology in a Menger space was introduced by B. Schweizer and A. Sklar. The base for the neighbourhoods of an element $x \in X$ is given by

$$
\left\{U_{x}(t, \epsilon) \subseteq X: t>0, \epsilon \in\right] 0,1[\}
$$

where

$$
U_{x}(t, \epsilon):=\left\{y \in X: F_{x, y}(t)>1-\epsilon\right\} .
$$

If the t-norm $\mathrm{T}$ satisfies the condition

$$
\sup \{T(a, a): a \in[0,1[\}=1,
$$

then the $(t, \epsilon)$-topology is metrizable.

The mapping $f: X \rightarrow X$ is said to be a contraction if there exists $r \in] 0,1[$ such that

$$
F_{f(x), f(y)}(r t) \geq F_{x, y}(t)
$$

for every $x, y \in X$ and $t \in \mathbb{R}_{+}$.

For example, if $(X, d)$ is a metric space and $G \in \Delta^{+}, G \neq H$, one defines

$$
F_{x, y}(t)=G\left(\frac{t}{d(x, y)}\right) \text { if } x \neq y
$$

and

$$
F_{x, y}(t)=H(t) \text { if } x=y .
$$

If $f: X \rightarrow X$ is a contraction with ratio $r$, then it is a contraction in Sehgal sense with the same ratio. Indeed, we have

$$
\begin{aligned}
F_{f(x), f(y)}(r t) & =G\left(\frac{r t}{d(f(x), f(y))}\right) \geq G\left(\frac{r t}{r d(x, y)}\right)= \\
& =F_{x, y}(t) \text { if } f(x) \neq f(y) \text { and } x \neq y,
\end{aligned}
$$




$$
\begin{gathered}
F_{f(x), f(y)}(r t)=\quad H(r t) \geq G\left(\frac{t}{d(x, y)}\right)=F_{x, y}(t) \text { if } x \neq y \text { and } f(x)=f(y), \\
F_{f(x), f(y)}(r t)=H(t)=F_{x, y}(t) \text { if } x=y .
\end{gathered}
$$

A sequence $\left(x_{n}\right)_{n \in \mathbb{N}}$ from $X$ is said to be fundamental if

$$
\lim _{n, m \rightarrow \infty} F_{x_{m}, x_{n}}(t)=1
$$

for all $t>0$.

The element $x \in X$ is called limit of the sequence $\left(x_{n}\right)_{n \in \mathbb{N}}$, and we write $\lim _{n \rightarrow \infty} x_{n}=x$ or $x_{n} \rightarrow x$, if $\lim _{n \rightarrow \infty} F_{x, x_{n}}(t)=1$ for all $t>0$.

A probabilistic metric (Menger) space is said to be complete if every fundamental sequence in that space is convergent.

For example, if $(X, d)$ is a metric space, then the metric $d$ induces a mapping $\mathcal{F}: X \times X \rightarrow \Delta^{+}$, where $\mathcal{F}(x, y)=F_{x, y}$ is defined by

$$
F_{x, y}(t)=H(t-d(x, y)), t \in \mathbb{R} .
$$

Moreover $(X, \mathcal{F}, M i n)$ is a Menger space. Bharucha-Reid and Sehgal show that $(X, \mathcal{F}, M i n)$ is complete if the metric $d$ is complete. The space $(X, \mathcal{F}, M i n)$ thus obtained is called the induced Menger space.

Theorem 1.1 ([8]). Every contraction mapping $f: X \rightarrow X$ on a complete Menger space $(X, \mathcal{F}, M i n)$ has a unique fixed point $x_{0}$. Moreover, $f^{n}(x) \rightarrow x_{0}$ for each $x \in X$.

Set

$$
\mathcal{D}^{+}=\left\{F \in \Delta^{+}: \sup _{t \in \mathbb{R}} F(t)=1\right\} .
$$

In the following we always suppose that $(X, \mathcal{F}, T)$ is a Menger space with $\mathcal{F}$ : $X \times X \rightarrow \mathcal{D}^{+}$and $\mathrm{T}$ is continuous.

Let $A$ be a nonempty subset of $X$.

The function $D_{A}: \mathbb{R} \rightarrow[0,1]$ defined by

$$
D_{A}(t):=\sup _{s<t} \inf _{x, y \in A} F_{x, y}(s)
$$

is called the probabilistic diameter of $A$.

It is easy to check that $\mathrm{D}_{A} \in \Delta^{+}$.

The set $A \subseteq X$ is probabilistic bounded if $D_{A} \in \mathcal{D}^{+}$.

Let $A$ and $B$ be nonempty subsets of $X$.

The probabilistic Hausdorff-Pompeiu distance between $A$ and $B$ is the function $F_{A, B}: \mathbb{R} \rightarrow[0,1]$ defined by

$$
F_{A, B}(t):=\sup _{s<t} T\left(\inf _{x \in A} \sup _{y \in B} F_{x, y}(s), \inf _{y \in B} \sup _{x \in A} F_{x, y}(s)\right) .
$$

The collection of nonempty closed bounded subsets of a Menger space is a Menger space also: 
Theorem $1.2([4])$. If $\mathcal{C}$ is a nonempty collection of nonempty closed bounded sets in a Menger space $(X, \mathcal{F}, T)$ with $T$ continuous, then $\left(\mathcal{C}, \mathcal{F}_{\mathcal{C}}, T\right)$ is also Menger space, where $\mathcal{F}_{\mathcal{C}}$ is defined by $\mathcal{F}_{\mathcal{C}}(A, B):=F_{A, B}$ for all $A, B \in \mathcal{C}$.

The next theorem shows the completeness of the Menger space obtained in the previous proposition for $T(a, b):=\operatorname{Min}:=\min \{a, b\}$.

Theorem $1.3([4])$. If $(X, \mathcal{F}$, Min) is a complete Menger space and $\mathcal{C}$ is the collection of all nonempty closed bounded subsets of $X$ in $(t, \epsilon)-$ topology, then $\left(\mathcal{C}, \mathcal{F}_{\mathcal{C}}\right.$, Min $)$ is also a complete Menger space.

The collection $\mathcal{K}$ of all nonempty compact subsets of $X$ form a complete Menger space if $(X, \mathcal{F}, M i n)$ is a complete Menger space.

Theorem 1.4 ([4]). Let $\mathcal{K}$ be the collection of all nonempty compact sets in the complete Menger space $(X, \mathcal{F}, M i n)$ and let $\mathcal{C}$ be the collection of all nonempty closed bounded subsets of $X$ in $(t, \epsilon)-$ topology. Then $\left(\mathcal{K}, \mathcal{F}_{\mathcal{K}}, M i n\right)$ is a closed subspace of $\left(\mathcal{C}, \mathcal{F}_{\mathcal{C}}, \operatorname{Min}\right)$.

If $(X, \mathcal{F}, M i n)$ is a complete Menger space and $\mathcal{K}$ is the collection of all nonempty compact subsets of $X$ in $(t, \epsilon)$ - topology, then $\left(\mathcal{K}, \mathcal{F}_{\mathcal{K}}, M i n\right)$ is also a complete Menger space.

\section{Invariant sets in probabilistic metric spaces}

We prove the existence and uniqueness of invariant sets in probabilistic metric spaces. This theorem generalize Hutchinson's theorem [3] on invariant sets.

Theorem $2.1([4])$. Let $(X, \mathcal{F}$, Min) be a Menger space and $\mathcal{C}$ be the collection of all nonempty closed bounded sets in $X$. Let $N \in \mathbb{N}, N \geq 1, f_{1}, \ldots, f_{N}: X \rightarrow X$ be contractions with ratios $\left.r_{1}, \ldots, r_{N} \in\right] 0,1[$ and let $\phi: \mathcal{C} \rightarrow \mathcal{C}$ be defined by

$$
\phi(E):=\cup_{i=1}^{N} f_{i}(E) .
$$

Then $\phi$ is a contraction.

Proof. Let $r=\max \left\{r_{i}, 1 \leq i \leq N\right\}$ and $A, B \in \mathcal{C}$. We shall show that

$$
F_{\phi(A), \phi(B)}(r t) \geq F_{A, B}(t)
$$

for all $t>0$.

For all $A, B \in \mathcal{C}$ and $s<t$, we have

$$
\begin{aligned}
& F_{\cup_{i=1}^{N} f_{i}(A), \cup_{i=1}^{N} f_{i}(B)}(r t) \geq \\
\geq & T\left(\inf _{u \in \cup_{i=1}^{N} f_{i}(A)} \sup _{v \in \cup_{i=1}^{N} f_{i}(B)} F_{u, v}(r s), \inf _{v \in \cup_{i=1}^{N} f_{i}(B)} \sup _{y \in \cup_{i=1}^{N} f_{i}(A)} F_{u, v}(r s)\right) .
\end{aligned}
$$


Let $i_{0}$ and $j_{0}$ be such that

$$
\begin{aligned}
\inf _{u \in \cup_{i=1}^{N} f_{i}(A)} \sup _{v \in \cup_{i=1}^{N} f_{i}(B)} F_{u, v}(r s) & =\inf _{u \in f_{i_{0}}(A)} \sup _{v \in \cup_{i=1}^{N} f_{i}(B)} F_{u, v}(r s) \geq \\
& \geq \inf _{u \in f_{i_{0}}(A)} \sup _{v \in f_{i_{0}}(B)} F_{u, v}(r s), \\
\inf _{v \in \cup_{i=1}^{N} f_{i}(B)} \sup _{u \in \cup_{i=1}^{N} f_{i}(A)} F_{u, v}(r s) & =\inf _{y \in f_{j_{0}}(B)} \sup _{u \in \cup_{i=1}^{N} f_{i}(A)} F_{u, v}(r s) \geq \\
& \geq \inf _{v \in f_{j_{0}}(B)} \sup _{u \in f_{j_{0}}(A)} F_{u, v}(r s) .
\end{aligned}
$$

Hence

$$
\begin{gathered}
F_{\cup_{i=1}^{N} f_{i}(A), \cup_{i=1}^{N} f_{i}(B)}(r t) \geq T\left(\inf _{u \in f_{i_{0}}(A)} \sup _{v \in f_{i_{0}}(B)} F_{u, v}(r s), \inf _{v \in f_{j_{0}}(B)} \sup _{u \in f_{j_{0}}(A)} F_{u, v}(r s)\right) \\
\geq T\left(\inf _{u \in f_{l_{0}}(A)} \sup _{v \in f_{l_{0}}(B)} F_{u, v}(r s), \inf _{v \in f_{l_{0}}(B)} \sup _{u \in f_{l_{0}}(A)} F_{u, v}(r s)\right)= \\
=T\left(\inf _{x \in A} \sup _{y \in B} F_{f_{l_{0}}(x), f_{l_{0}}(y)}(r s), \inf _{y \in B} \sup _{x \in A} F_{f_{l_{0}}(x), f_{l_{0}}(y)}(r s)\right) \geq \\
\geq T\left(\inf _{x \in A} \sup _{y \in B} F_{x, y}(s), \inf _{y \in B} \sup _{x \in A} F_{x, y}(s)\right),
\end{gathered}
$$

where $l_{0}=i_{0}$ if

$$
\inf _{u \in f_{i_{0}}(A)} \sup _{v \in f_{i_{0}}(B)} F_{u, v}(r s) \leq \inf _{v \in f_{j_{0}}(B)} \sup _{u \in f_{j_{0}}(A)} F_{u, v}(r s)
$$

and $l_{0}=j_{0}$ else. Therefore we have (2.1).

Theorem $2.2([4])$. Let $\left(X, \mathcal{F}\right.$, Min) be a complete Menger space and let $f_{1}, \ldots, f_{N}$ : $X \rightarrow X$ be contractions with ratios $\left.r_{1}, \ldots, r_{N} \in\right] 0,1[$, respectively. Then there exists a nonempty compact subset $K$ of $X$ such that

$$
f_{1}(K) \cup \ldots \cup f_{N}(K)=K \text {. }
$$

Moreover, the set $K$ with this property is unique in the space of all nonempty closed bounded sets in $X$.

Proof. By Proposition 2.1 the function $\phi: \mathcal{C} \rightarrow \mathcal{C}$ defined by

$$
\phi(E)=\cup_{i=1}^{N} f_{i}(E)
$$

is a contraction, and by Proposition $1.3\left(\mathcal{C}, \mathcal{F}_{\mathcal{C}}, M i n\right)$ is a complete Menger space. Then, by Proposition 1.1 there is a unique set $K$ in $\mathcal{C}$ such that $\phi(K)=K$. Moreover, we have $\lim _{n \rightarrow+\infty} \phi^{n}\left(K_{0}\right)=K$ for any $K_{0} \in \mathcal{K}$. Thus, by Proposition 1.4 the set $K$ must be in $\mathcal{K}$. 


\section{Application to quicksort algorithm}

Let $\sum$ be the nonempty alphabet and $\sum^{\infty}$ be the set of all sequences over $\sum$ (finite or infinite). Suppose $\emptyset \in \sum$. Denote by $\prec$ the prefix order on $\sum^{\infty}$. For each $x \in \sum^{\infty}$ denote $l(x)$ the lenght of $x$. For $x, y \in \sum^{\infty}$ let $x \wedge y$ the common prefix of $x$ and $y$. Let the function $d_{\prec}: \sum^{\infty} \times \sum^{\infty} \rightarrow \mathbb{R}$ defined by

$$
\begin{gathered}
d_{\prec}(x, y)=0 \text {, if } x \prec y \\
d_{\prec}(x, y)=2^{-l(x \wedge y)}, \text { otherwise. }
\end{gathered}
$$

Let $F_{x, y}(t)=\left\{\begin{array}{cc}0, & t=0 \\ \frac{t}{t+k^{-l(x \wedge y)}}, & t>0, x \text { not prefix of } y, k>1 \\ 1, & x \text { prefix of } y\end{array}\right.$

Using the complexity analysis given in [2] we will get the following recurrence equation:

$$
T(1)=0
$$

and

$$
T(n)=\frac{2(n-1)}{n}+\frac{n+1}{n} T(n-1), n \geq 2 .
$$

We associate to $T$ the functionals $f_{k}: \sum^{\infty} \rightarrow \sum^{\infty}$ such that

$$
\left(f_{k}(x)\right)_{1}=T(1)
$$

and

$$
\left(f_{k}(x)\right)_{n}=\frac{2(n-1)}{n}+\frac{n+1}{n} x_{n-1},
$$

for $k=2,3, \ldots, m . f_{k}$ will be a contraction with ratio $\frac{1}{k}$.

Using theorem 2.2 for the contractions $f_{2}, \ldots, f_{m}$ starting from the $\left(\sum^{\infty}, \mathcal{F}\right.$, Min $)$ Menger space, we will get the invariant set of solutions of the reccurence equation. For a fixed $k$ this is the unique solution of $\mathrm{T}$.

\section{References}

[1] J.A.Fill, S.Janson: A characterization of the Set of Fixed Points of the Quicksort Transformation, Elect. Comm. in Probab. 5(2000) 77-84.

[2] P.Flajole: Analytic analysis of algoritms in Lectures Notes in Computer Science, vol 623, Springer, 1992, 186-210.

[3] J.E.Hutchinson: Fractals and Self Similarity, Indiana University Mathematics Journal, 30 (1981), no.5, 713-747.

[4] J.Kolumbán, A.Soós: Invariant sets in Menger spaces, Studia Univ. "BabesBolyai", Mathematica, 43, 2 (1998), 39-48.

[5] K.Menger: Statistical Metrics, Proc.Nat. Acad. of Sci.,U.S.A. 28 (1942), 535-537. 
[6] S.Romaguera, A. Sapena, P. Tirano: The Banach fixed point theorem in fuzzy quasi-metric spaces with application to the domain of words, Topology and its Applications 154 (2007), 2196-2203.

[7] B.Schweizer, A.Sklar: Probabilistic Metric Spaces, North Holland, New-York, Amsterdam, Oxford, 1983.

[8] V.M.Sengal: Some Fixed Point Theorems in Functional Analysis and Probability, Ph.D.Thesis, Wayne State University, 1966. 